fish, \&c., I cannot help thinking that Lilienthal's central and superposed aeroplanes were a mistake; and that instead of that type, while the weight must be central, the sustaining aeroplanes should, like the birds, have great lateral extension.

You will observe in the diagram that the wing planes can each be divided into two portions, having quite distinct functions. The outer extremities are the sustaining aeroplanes, marked by the arrows, while the inner portion of each wing, $A$ to $W$, is that which assists the bird when it is alighting, by offering a fixed passive resistance to a fall when the speed is slackened down. $W$ is the central weight.

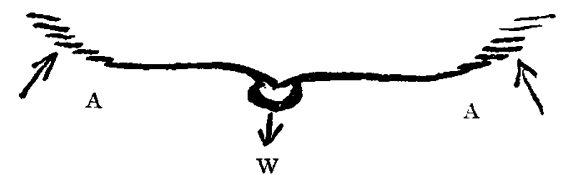

Observe also that in the bird, the sustaining mechanism is so far structurally subdivided that the loss of a primary feather is not fatal to flight; each primary lies, and acts, in a distinct plane, and has its attachment distinct from the others.

Now, it seems to me that Mr. Maxim's central aeroplane and twin screws, situated so far apart, are hardly a safe plan, for if accident happen to one screw, the other must at once stop, and the whole thing, nolens volens, come down.

It is not like the twin-screw steamer, where the water sustains the hull, and progress by one screw is still possible. In the aerial ship translation is the support, and it only.

In the bird, when sailing, we see no screw at work; the aeroplanes are there plain enough, lifting the I6-pound bird higher and higher as we watch it; but propeller there is none.

This propulsion, as I before stated, must be got from an outside source. The bird can only soar in a wind, and then, to rise, must go in spirals, passing to leeward a little at each lap. Of course the wing planes are not horizontal, but inclined thus in

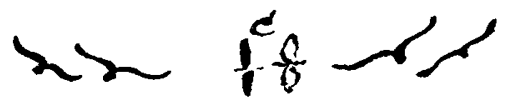

passing round the centre of spiral $\mathrm{C}$; and there is necessarily great centripetal reaction at such a high speed of translation as fifty or sixty miles per hour.

I think Mr. Maxim will find the bird arrangement of aeroplanes to weight, and a central screw, the best and safest. If a large central overhead aeroplane is needed, it would be for safety in alighting only.

Sibsagar, Asam, December I3, 1896.

S. E. PEAL.

\section{OSMOTIC PRESSURE.}

I N last week's NATURF, Lord Rayleigh gave, for an involatile liquid, a rigorous and clear proof of "the Central Theorem" of osmotics. But this theorem, though highly interesting in itself, is not, so far as I can see, useful as a guide for experiment. Consider for example the typical cases of sugar, and of common salt, dissolved in water.

If water were absolutely non-volatile, the osmotic pressure of each solution against an ideal semi-permeable membrane separating it from pure water, would, according to the theorem, be equal to the calculable pressure of the ideal gas of the dissolved substance supposed alone in the space occupied by the solution. This would be true whatever be the molecular groupiny of the sugar or of the salt in the solution. It is believed that experiment has verified the theorem, extended to volatile solvents, as approximately true for sugar and several other substances of organic origin, and of highly complex atomic structure; but has proved it to vastly under-estimate the osmotic pressure for common salt and many other substances of similarly simple composition.

KELVIN.

Belfast, January 19.

\section{On Osmotic Pressure against an Ideal Semi- PERMEABLE MEMBRANE. ${ }^{1}$}

To approach the subject of osmotic pressure against an ideal impermeable membrane by the easiest way, consider first a vessel filled with any particular fluid divided into two parts, $\mathrm{A}$ and $\mathrm{B}$, by an ideal surface, MM. Let a certain number of individual molecules of the fluid in $\mathrm{A}$, any one of which we shall call $\mathrm{D}$ (the dissolved substance), be endowed with the property that they cannot cross the surface MM (the semi-permeable membrane); but let them continue to be in other respects exactly similar to every other molecule of the fluid in A, and to all the molecules of the fluid in $B$, any one of which we shall call $S$ (the solvent), each of which can freely cross the membrane. Suppose now the con. taining vessel and the dividing membrane all perfectly rigid. ${ }^{2}$ Let the apparatus be left to itself for so long time that no further change is perceptible in the progress towards final equilibrium of temperature and pressure. The pressures in $\mathrm{A}$ and $\mathrm{B}$ will be exactly the same as they would be with the same densities of the fluid if MM were perfectly impermeable, and all the molecules of the fluid were homogeneous in all qualities; and MM will be pressed on one side only, the side next $A$, with a force equal to the excess of the pressure in $\mathrm{A}$ above the pressure in $B$, and due solely to the impacts of I) molecules striking it and rebounding from it.

If now, for a moment, we suppose the fluid to be "perfect gas," we should find the pressure on MM to be equal to that which would be produced by the $\mathrm{D}$ molecules if they were alone in the space $\mathrm{A}$; and this is, in fact, very approximately what the osmotic pressure would be with two ordinary gases at moderate pressures, one of which is confined to the space $A$ by a membrane freely permeable by the other. On this supposition the number of the $S$ molecules per unit bulk would be the same on the two sides of the membrane. And if, for example, there are Iooo $S$ molecules to one $D$ molecule in the space $A$, the pressure on the piston $P$ would be roo I times the osmotic pressure, and on Q rooo times the osmotic pressure. But if the fluid be "liquid" on both sides of the membrane, we may annul the pressure on $Q$ and reduce the pressure on $\mathrm{P}$ to equality with the osmotic pressure, by placing the apparatus under the receiver of an air-pump, or by pulling $Q$ outwards with a force equal and opposite to the atmospheric pressure on it. When we do this, the annulment of the integral pressure of the liquid on the piston $Q$ is effected through balancing by attraction, of pressure due

3. Communicated to the Royal Society of Edinburgh, January 18, by Lord Kelvin.

2 In the drawing, the vessel is represented by a cylinder closed at each end by a piston to facilitate the consideration of what will happen if, instead of supposing it rigid, any arbitrary condition as to the pressures on the two of supposing it rigid, any arbitrary
sides of the membrane be imposed.

No. I 42 I, VOL. 55$]$ 